\title{
An Adaptive RR Interval Detection Algorithm Based on Species Recognition
}

\author{
Dan $\mathrm{Li}^{1}$, Wu Huang ${ }^{2}$, Guobiao Xu${ }^{3}$, Tao Zhang ${ }^{1}$, Zhonghui Jiang ${ }^{1}$, Defu Cheng ${ }^{1}$ \\ ${ }^{1}$ Chengdu Techman Software Co., Ltd, Chengdu, China. \\ 2 Sichuan University, Chengdu, China. \\ ${ }^{3}$ China Civil Aviation Flight University, Chengdu, China. \\ * Corresponding author. Tel.:13088058715; email: tmezl@126.com \\ Manuscript submitted July 4, 2019; accepted September 19, 2019. \\ doi: 10.17706/ijcee.2019.11.4.180-191
}

\begin{abstract}
Many algorithms require prior information about test subjects for heart rate (HR) detection. In this paper, we propose a novel algorithm for detecting the HR of previously unknown species based on the Pan-Tompkins (PT) algorithm without a priori knowledge. In this improved PT algorithm, some parameters that need to be predefined can be adaptively adjusted according to the recognition of previous species. In the recognition step, a clustering algorithm is applied to obtain the rough RR intervals, and a decision tree is applied for species recognition by using two features: the rough RR intervals and the proportion of the ECG power of frequency that is less than $5 \mathrm{~Hz}$. The accuracy and the Kappa of species classification in the recognition step can reach $93.15 \%$ and $90.23 \%$, respectively. In the HR detection step, the improved PT algorithm is used to precisely detect the HR of rats, mice, humans, frogs and rabbits, and the results show that this method has good performance. In particular, we apply the proposed algorithm to test some ECG signals in humans from the MIT-BIH database. The results show that the accuracy of the proposed algorithm for detection of HR in humans reaches $99.71 \%$.
\end{abstract}

Key words: Clustering algorithm, decision tree, pan-tompkins algorithm, RR-intervals, heart rate.

\section{Introduction}

As an important physiological index, heart rate (HR) is closely related to many biological features, such as body mass and the lifespan of mammals [1], [2]. For humans, HR is a particularly useful clinical variable that can indicate some serious diseases, including cardiovascular diseases and cancers [3], [4]. To measure HR, the electrocardiogram (ECG) has been widely used for many years, and the feasibility of some novel ECG-based methods for non-contact HR measurement have recently been explored [5]. Additionally, non-contact technologies for HR measurement can be achieved by analyzing videos of faces or time-lapse images from a CCD camera [6], [7].

In ECG-based detection methods, some useful features of ECG signals need to be extracted for HR measurement. Compared to other features, the RR intervals may be more feasible to extract in the presence of noise. To rapidly and accurately detect RR intervals, many algorithms for detecting the QRS complex have been investigated, including filter-based algorithms [8]-[13], wavelet-based algorithms [14], [15] and neural networks [16], [17]. In addition, because of the importance of QRS detection in studies and other applications, more algorithms have been reviewed in [18]. Wavelet-based algorithms and neural networks have good performance in QRS detection, but it is difficult to apply them in real time because of their 
complexity [14]-[17]. For filter-based algorithms, adaptive filters have been widely studied and used for QRS detection. However, some require prior information for test subjects to set the parameters [8], [11].

In this work, the goal is to find a simple method for detecting the HR of previously unknown species without knowing prior information about them. First, the species are recognized by using a decision tree in which the rough RR interval is one of the features extracted with a clustering algorithm. Second, the HR of species is detected by using an improved Pan-Tompkins (PT) algorithm, where the parameters are determined from the recognition results for previous species. For evaluation, the proposed algorithm is applied to detect the HR of rats, mice, humans, frogs and rabbits, and the ECG signals were collected using the BL-420N (information) biological signal acquisition and processing system produced by Chengdu Techman Software Co., Ltd. Finally, the proposed algorithm is used to test some ECG signals from humans included in the MIT-BIH database.

\section{Methods}

The procedure for detecting the HR of diverse species by using the proposed algorithm is shown in Fig. 1. In the signal preprocessing step, adaptive filters are used to remove the baseline wander and the power-line interference, and the Kalman filter is used to further reduce noise disturbance. These filters do not require prior knowledge about the test species. Next, the square of the first derivative of the filtered ECG signals is calculated for species identification and HR detection, and a restriction function is used on the processed signals to limit the excessive values. It is worth mentioning that many algorithms for accurate QRS detection require prior knowledge of test subjects, and with this approach, we only roughly calculate the RR intervals as a feature without any prior knowledge. Therefore, in the feature extraction step, the rough RR intervals are extracted as the main feature for species recognition, and the R peak is obtained by using a clustering algorithm. In addition, to improve the recognition performance, another feature, which is the power proportion of frequencies less than $5 \mathrm{~Hz}$, is considered. In the recognition step, the species are predicted by a decision tree, where the two extracted features are used. In the last step, the HR is detected with an improved PT algorithm, in which the detected location is determined by the rough RR intervals from the results of feature extraction and the chosen empirical values from the results of species recognition.

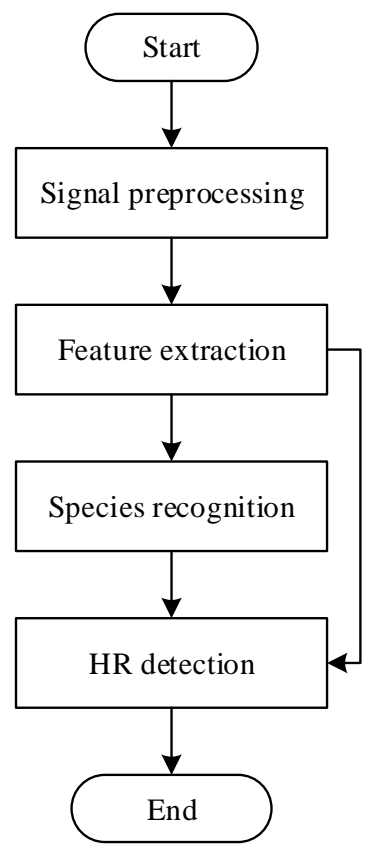

Fig. 1. Flow chart of the proposed HR detection algorithm for diverse species. 


\subsection{Preprocessing}

By using the BL-420N, we collected 97 segments of ECG data from rats, mice, frogs, humans and rabbits for our research. The sampling rate of each species is $2000 \mathrm{~Hz}$. In the preprocessing step, each processed segment should include at least two R peaks. In this study, the length of each selected ECG segment is between 5-10 s, and the length is sufficient to meet the required conditions. In general, ECG signals are susceptible to disturbances, such as baseline drift and power frequency disturbance [15]. Some filters require prior knowledge of ECG signals for setting parameters, such as the cut-off frequency and window width [11], [19]-[21]. For interpreting ECG signals without any prior knowledge about them, it is difficult to use these filters. Therefore, we just filter noise according to the noise characteristics. To remove the baseline drift and the power frequency disturbance, we choose adaptive filters whose reference signals are constant 1 and sine/cosine signals of $50 \mathrm{~Hz}$, respectively [22], [23]. Next, a Kalman filter is used to reduce other kinds of noise disturbance.

After filtering, the square of first derivative of filtered ECG signals is calculated. We search all the local maximum points on the square of first derivative of the filtered ECG signals for locating R peaks. Empirically the local maximum points corresponding to the $\mathrm{R}$ wave are much higher than other local maximum points. However, among the local maximum points corresponding to the $\mathrm{R}$ wave, some would be much higher than the rest. Therefore, to effectively use a clustering algorithm to search for $\mathrm{R}$ peaks, the amplitude of excessively high points should be limited. In this paper, we use a restriction function expressed as

$$
A_{\text {cor }}=a \log \left(a^{-1} A_{\text {ori }}+1\right)
$$

where $A_{\text {cor }}$ and $A_{\text {ori }}$ denote the corrected amplitude and original amplitude, respectively. Equation 1 implies that the restriction becomes obvious as a decreases. In this study, $a$ is set as the global maximum amplitude of the processed ECG signal segment. Consequently, the global maximum amplitude of the corrected curve is reduced to 0.69 times that of the original curve. However, in practice, $a$ can be properly adjusted according to the performance. If no restriction is required, a should be a sufficiently large number. In addition, as the amplitude decreases, this restriction is increasingly obscure. Although the process would shorten the gap between the local maximum points that corresponds to $\mathrm{R}$ waves and other irrelevant maximum points, observation shows that the gap is still obvious after correction. The curve of Eq.1 is shown in Fig. 2.

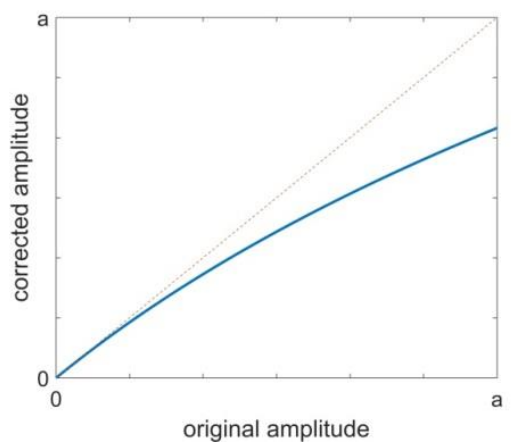

Fig. 2. Curve of the corrected amplitude (blue solid line) in which the red dashed line is the reference line of the original amplitude. The curves show that a larger amplitude for ECG signals leads to more change.

\subsection{Feature Extraction}

In this step, we extract two features from ECG segments for species recognition: the rough RR interval and the power proportion of frequencies less than $5 \mathrm{~Hz}$. Without excess accuracy, the RR interval is roughly obtained by using a clustering algorithm and can be a good feature for species recognition. The clustering 
algorithm divides all the local maximum points into two classes, and the class with the larger mean is the target class. The RR intervals are obtained by calculating the time intervals between two neighbouring local maximum points in the target class. The reason for why we use rough RR intervals rather than accurate RR intervals as the main feature is to avoid using prior knowledge. After classifying all the local maximum points with a clustering algorithm, we need to screen the target points, which are too close to each other. In fact, if there is more than one target point located in the time interval of $25 \mathrm{~ms}$, the point with the maximum amplitude should serve as the targeted point. For this reason, the HR of the test species should be less than $2400 \mathrm{bpm}$. Indeed, this method can effectively select the points corresponding to R waves, as shown in Fig. 3.

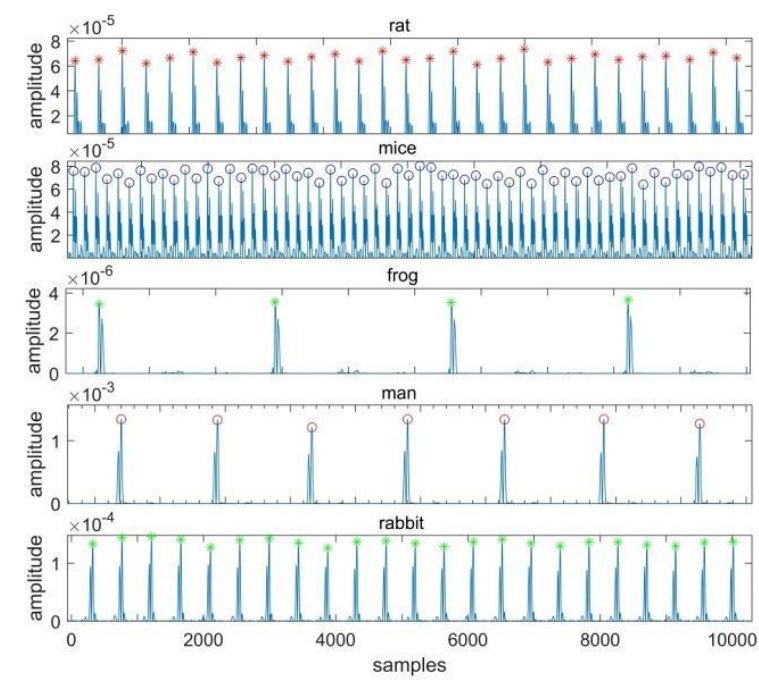

Fig. 3. R peaks of ECG signal segments from rats, mice, frogs, humans and rabbits predicted by a clustering algorithm.

The statistics of values for the two features are shown in Fig. 4. In Fig. 4(a), the rough RR intervals more accurately correspond to the HR of five species. Moreover, the values of the feature for five species have distinct distributions. However, the ranges of these rough RR intervals for different species still have overlaps, which may cause error recognition. For example, the overlap between the rough RR intervals of rats and rabbits is obvious because their HRs are similar to each other. Therefore, we add another feature to reduce errors, and those values are shown in Fig. 4(b). This feature is not as good as the rough RR intervals, but it can enhance the differences between five species, as shown in Fig. 4(c).
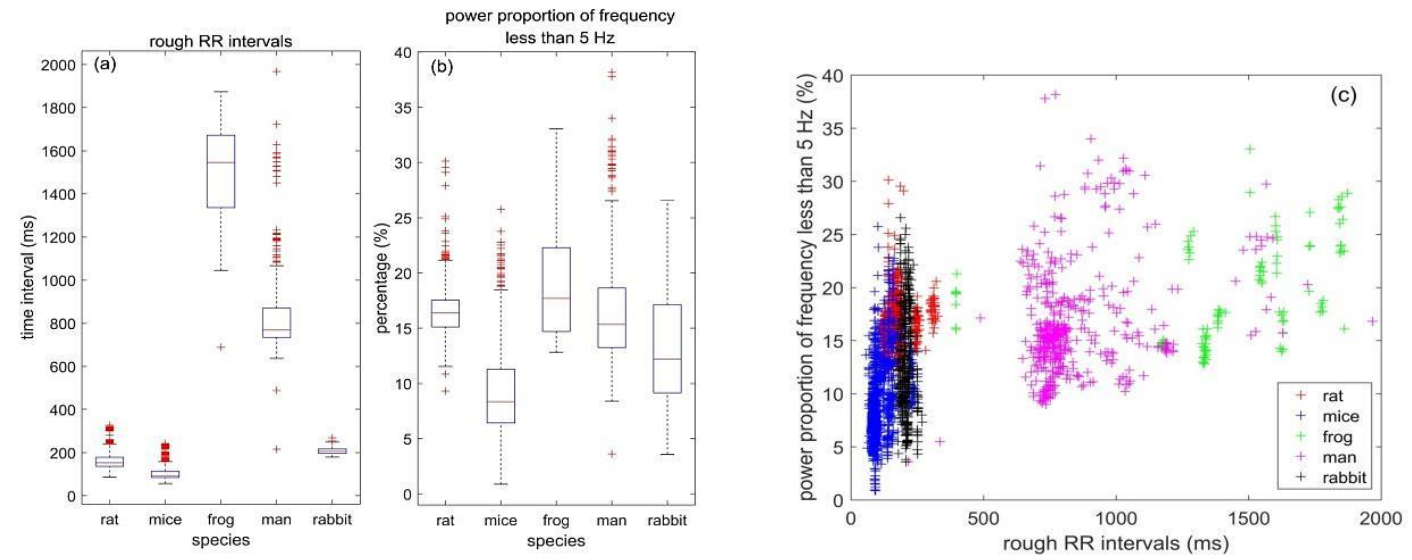

Fig. 4. (a) The box plot of rough RR intervals in relation to five species. (b) The box plot of the power proportion of frequencies less than $5 \mathrm{~Hz}$ in relation to five species. (c) Scatter plot of rough RR intervals and power proportion of frequencies less than $5 \mathrm{~Hz}$ in relation to five species. 


\subsection{Species Recognition}

In this step, we use a decision tree to predict the species type using 3310 samples. These samples are randomly divided into two parts. One part is the training data $(70 \%)$, and another part is the test data (30\%). To evaluate the species recognition performance with multiple aspects, we calculate the sensitivity and the precision, which are widely used as the evaluation indexes. The sensitivity can be calculated by

$$
R=\frac{T P}{T P+F N}
$$

where $T P$ denotes the true positive and $F N$ denotes the false negative. The precision can be calculated with

$$
P=\frac{T P}{T P+F P}
$$

where FP denotes the false positive.

The values of the sensitivity and the precision are obtained by running the program 100 times, as shown in Fig. 5. These box plots in Fig. 5 show how well the method predicts those species type. Fig. 5(a) shows that the mean values of sensitivity for the five species are over $92 \%$, and the maximum value of sensitivity for frogs and humans, in particular, can reach 100\%. Fig. 5(b) shows that the mean values of precision for rats, frogs and rabbits are between $80 \%$ and $90 \%$, and the values for humans and mice approach $100 \%$. It is known that the sensitivity and the precision are mutually restrictive, but here, both of them are comparably ideal for species recognition. Additionally, the sensitivity and the precision as well as the accuracy and the Kappa are calculated, which reach $93.15 \%$ and $90.23 \%$, respectively.
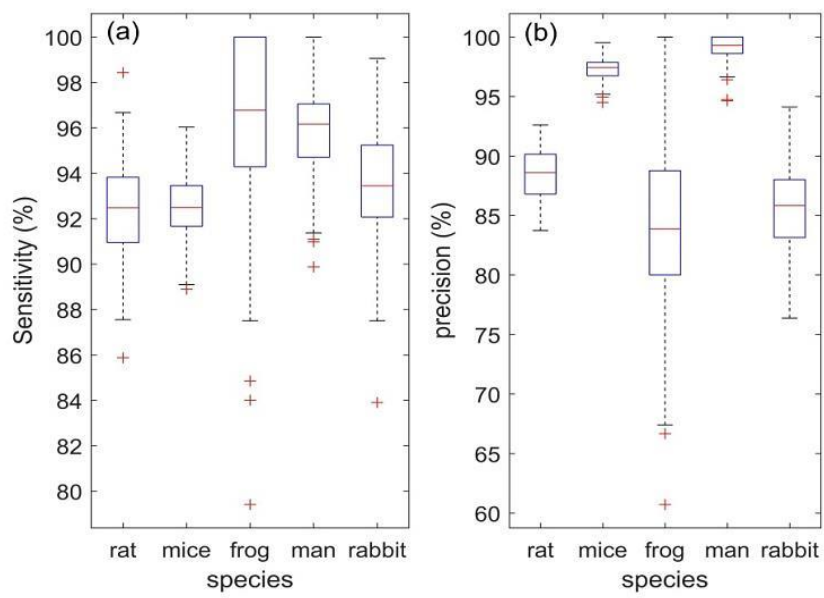

Fig. 5. Sensitivity (left) and precision (right) of species prediction using a decision tree where the values of two features in Fig. 4 are used as the training data (70\%) and test data (30\%). The decision tree is run 100 times, and the training data and the test data are randomly selected each time.

\subsection{HR Detection with the Improved PT Algorithm}

The rough RR intervals obtained in the feature extraction step would not be the ideal parameters for HR prediction. Moreover, it is difficult to directly detect the RR intervals from ECG signals by using the traditional PT algorithm because the width of the sliding window and the detected location cannot be set without some prior knowledge [11]. Therefore, in this step, we develop an improved PT algorithm for species HR detection with parameters that can be set adaptively according the recognition results for 
previous species.

In the traditional PT algorithm, the width of the sliding window is set according to the width of the QRS complex of the test subject, and the time interval of detection is also predefined. Therefore, when measurements for the test subject are unknown, these parameters cannot be set. In the improved PT algorithm, the width of the sliding window is set by an empirical value according to the results for species recognition. The empirical values are listed in Table 1 . For the detected location, the time interval between the detection start point and the last detection termination point is set according to the width of the QRS complex and the rough RR interval each time.

Table 1. Sliding Window Width for Various Species

\begin{tabular}{|c|c|}
\hline Species Category & Sliding Window Width (Samples) \\
\hline Human & 200 \\
\hline Frog & 300 \\
\hline Rabbit & 240 \\
\hline Rat & 171 \\
\hline Mice & 150 \\
\hline
\end{tabular}

\section{Results}

\subsection{Animal HR Detection}

The proposed algorithm is developed on a MATLAB workspace to process the ECG signal segments collected by the BL-420N (information) biological signal acquisition and processing system with a sampling rate of $2000 \mathrm{~Hz}$.

The performance of the proposed algorithm on HR detection for rats, mice, frogs and rabbits is shown In Fig. 6. From Figs. 6(a) to 6(d), it can be seen that the four animals have very different HRs. However, Figs. 6(a) to 6(d) show that the proposed algorithm has good performance on HR detection for the four animals. The reason for the good performance is that when the PT algorithm is used, the time interval of detection is adaptively adjusted by the rough RR intervals, and the width of the sliding window is adjusted by the QRS width according to Table 1. This method can detect the HR of animals in real time.
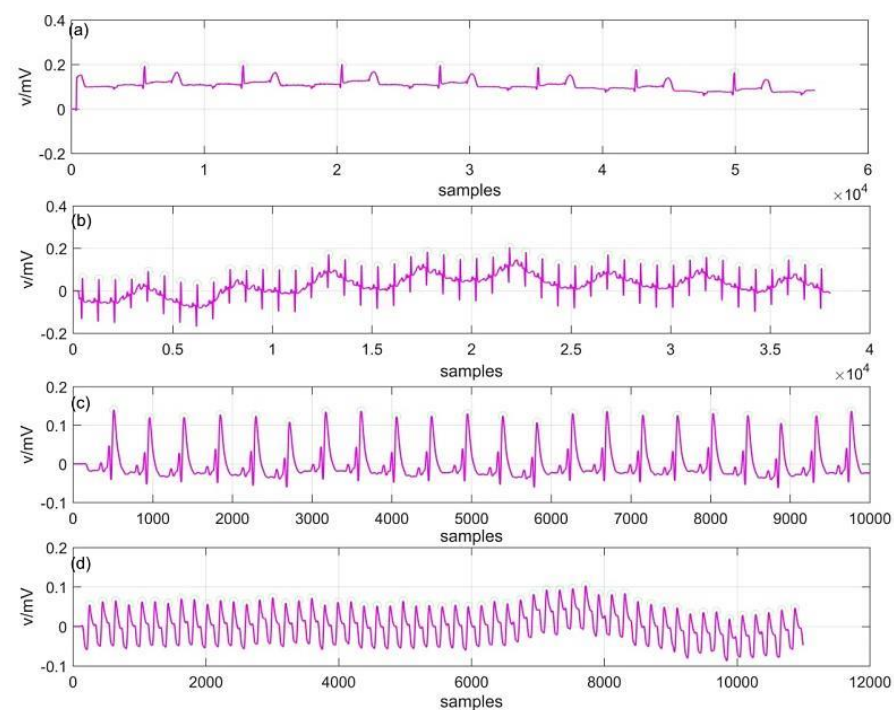

Fig. 6. Detection of R peaks with the proposed algorithm for (a) frogs, (b) rabbits, (c) rats and (d) mice. 


\subsection{Human HR Detection}

In Fig. 7, the proposed algorithm is compared with the standard PT algorithm for human HR detection. In Fig. 7(a), the standard PT algorithm misjudges R peaks on the horizontal straight line for the front segment of the ECG signal. In contrast, the proposed algorithm can recognize and eliminate the meaningless signal segments and perfectly detect the R peak, as shown in Fig. 7(b). Moreover, for the meaningful ECG signal segments, the proposed algorithm performs better than the standard PT algorithm. The comparisons in Fig. 7(c) and Fig. 7(d) show that the proposed algorithm can perfectly detect the real $\mathrm{R}$ peaks, but some omissions and errors appear when using the PT algorithm for HR detection.
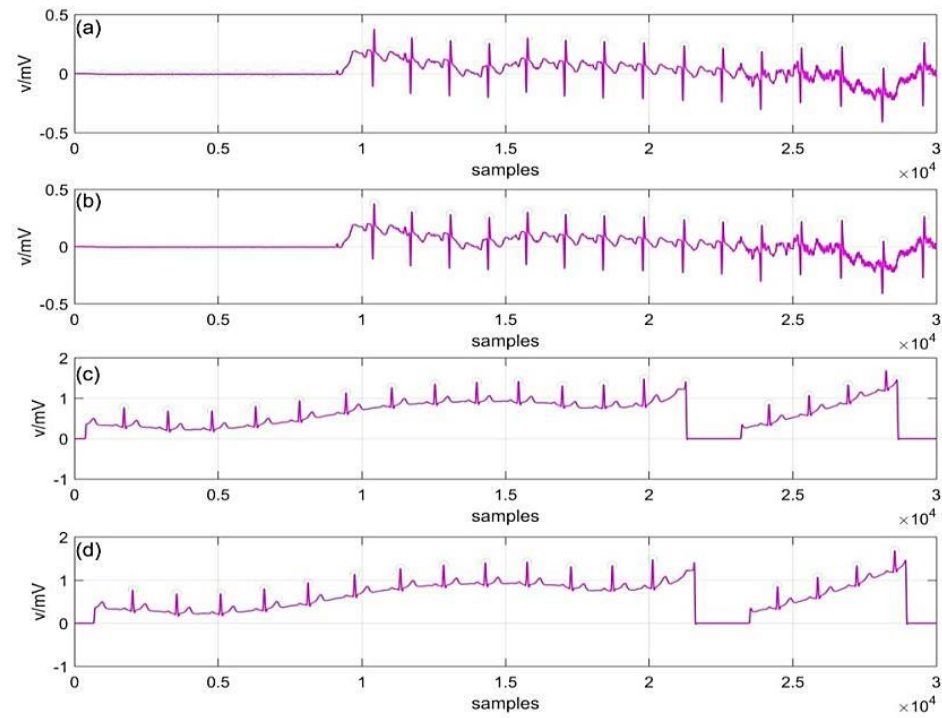

Fig. 7. Comparison between the proposed algorithm and the standard PT algorithm for detection of R peaks based on the ECG signals of humans. Detection of R peaks for humans with the standard PT algorithm is shown in (a) and (c), and detection of R peaks for humans with the proposed algorithm is shown in (b) and (d).

To further evaluate the performance of the proposed algorithm, we select 43 representative ECG signals from the MIT-BIH standard ECG database for testing. In addition to the sensitivity and the precision in Eqs.2 and 3 , we select the error rate as one of the evaluation indexes, which is expressed as

$$
E R=1-\frac{T P}{T P+F P+T N+F N}
$$

The testing results are listed in Table 2. Table 2 shows that the average accuracy rate can reach 99.71\%, which meets the clinical standard (99\% [24]). For some ECG signals, the accuracy rates can even be better, such as $99.96 \%$ for No. 100, No. 109 and No. 112. However, for No. 114 signal, the failed detection is very high (5.58\%), which may be caused by the noise interference and the variation of waveforms. ECG signals segments disturbed by a mass of noise would cause detection error and would need to be discarded in HR detection. Furthermore, we compare the performance of the proposed algorithm (PM) with the PT algorithm [11] and a wavelet transform algorithm (WT) [25] for human HR detection. The failed detection of the proposed algorithm is approximately half of that for the PT algorithm. Although the failed detection of the proposed algorithm is approximately two times higher than that of the wavelet transform algorithm, the proposed algorithm runs faster than the wavelet transform because it does not detect the modulus maxima and the Lipschitz exponent. 
Table 2. Results of HR Detection are Tested Using the PM, WT and PT Algorithm for Comparing the Error Rates

\begin{tabular}{|c|c|c|c|c|c|c|}
\hline \multirow{2}{*}{ Tape(No.) } & \multirow{2}{*}{$\begin{array}{c}\text { Total } \\
\text { (No.Beats) }\end{array}$} & \multicolumn{3}{|c|}{ Error rate $(\%)$} & \multirow{2}{*}{ Accuracy (\%) } & \multirow{2}{*}{ Sensitivity (\%) } \\
\hline & & PM & PT & WT & & \\
\hline 100 & 2273 & 0.04 & 0 & 0 & 99.96 & 99.96 \\
\hline 101 & 1865 & 0 & 0.43 & 0 & 100 & 100 \\
\hline 102 & 2187 & 0.09 & 0 & 0.11 & 99.91 & 99.95 \\
\hline 103 & 2083 & 0 & 0 & 0 & 100 & 100 \\
\hline 104 & 2229 & 0 & 0.04 & 0.45 & 100 & 100 \\
\hline 105 & 2572 & 0.93 & 3.46 & 1.09 & 99.07 & 99.92 \\
\hline 106 & 2027 & 0.59 & 0.05 & 0.25 & 99.41 & 100 \\
\hline 107 & 2137 & 0.65 & 0.09 & 0 & 99.35 & 100 \\
\hline 108 & 1763 & 0.17 & 12.54 & 1.59 & 99.83 & 100 \\
\hline 109 & 2532 & 0.04 & 0.04 & 0 & 99.96 & 100 \\
\hline 111 & 2124 & 0.05 & 0.05 & 0.09 & 99.95 & 100 \\
\hline 112 & 2538 & 0.04 & 0.04 & 0.12 & 99.96 & 99.96 \\
\hline 113 & 1794 & 0 & 0 & 0.11 & 100 & 100 \\
\hline 114 & 1879 & 5.58 & 1.06 & 0 & 94.42 & 98.58 \\
\hline 115 & 1952 & 0 & 0 & 0 & 100 & 100 \\
\hline 116 & 2394 & 0.21 & 1.04 & 0.04 & 99.79 & 99.83 \\
\hline 117 & 1535 & 0.13 & 0.13 & 0.07 & 99.87 & 99.93 \\
\hline 118 & 2278 & 0 & 0.04 & 0.04 & 100 & 100 \\
\hline 119 & 1987 & 0 & 0.05 & 0.05 & 100 & 100 \\
\hline 121 & 1863 & 0.11 & 0.59 & 0.16 & 99.89 & 99.95 \\
\hline 122 & 2476 & 0.04 & 0.08 & 0 & 99.96 & 99.96 \\
\hline 123 & 1518 & 0.06 & 0 & 0 & 99.94 & 99.93 \\
\hline 124 & 1619 & 0.12 & 0 & 0 & 99.88 & 100 \\
\hline 200 & 2601 & 0.23 & 0.35 & 0.04 & 99.77 & 99.96 \\
\hline 201 & 1963 & 0.76 & 0.51 & 0.66 & 99.24 & 99.95 \\
\hline 202 & 2136 & 0.12 & 0.19 & 0.05 & 99.91 & 100 \\
\hline 205 & 2656 & 0.48 & 0.08 & 0.04 & 99.52 & 99.89 \\
\hline 208 & 2956 & 0.64 & 0.6 & 0.14 & 99.36 & 99.46 \\
\hline 209 & 3004 & 0 & 0.1 & 0 & 100 & 100 \\
\hline 212 & 2747 & 0 & 0 & 0 & 100 & 100 \\
\hline 213 & 3251 & 0.15 & 0.09 & 0 & 99.85 & 99.88 \\
\hline 214 & 2262 & 0.13 & 0.26 & 0.87 & 99.87 & 99.96 \\
\hline 215 & 3363 & 0.12 & 0.03 & 0.4 & 99.88 & 99.88 \\
\hline 217 & 2208 & 0.23 & 0.45 & 0.09 & 99.77 & 99.95 \\
\hline 220 & 2048 & 0.1 & 0 & 0 & 99.9 & 99.9 \\
\hline 221 & 2427 & 0.2 & 0.29 & 0.08 & 99.8 & 99.92 \\
\hline 223 & 2605 & 0.04 & 0.04 & 0.08 & 99.96 & 100 \\
\hline 228 & 2053 & 0.68 & 1.46 & 0.49 & 99.32 & 99.85 \\
\hline 230 & 2256 & 0 & 0.04 & 0 & 100 & 100 \\
\hline 231 & 1570 & 0 & 0 & 0 & 100 & 100 \\
\hline 232 & 1780 & 0.11 & 0.39 & 0 & 99.89 & 100 \\
\hline 233 & 3079 & 0.58 & 0.03 & 0 & 99.42 & 99.61 \\
\hline 234 & 2753 & 0 & 0 & 0 & 100 & 100 \\
\hline 43 & 97343 & 0.29 & 0.48 & 0.14 & 99.71 & 99.91 \\
\hline
\end{tabular}




\section{Discussion}

HR detection methods have been studied for many years. However, most of them involve humans rather than animals. For various species, the HRs are very different, and their ECG curves are complicated. Therefore, some QRS detection methods developed for humans are difficult to apply to animals because some parameters in these methods, which must be predefined according to certain features of ECG signals from humans, cannot be appropriately set. A method used for detecting the QRS complex of ECG signals from animals should include common features of ECG signals from different species. Through observation, we found that the local maximum points on the square of the first derivative of the filtered ECG signals corresponding to the $\mathrm{R}$ wave are much higher than the other local maximum points, and thus, a clustering algorithm was chosen to search the target points.

The goal of the work is to detect the HR of unknown species based on ECG signals, which implies that some prior information about the species cannot be used. Some QRS predictors require knowing the rough periods of ECG signals, but it is difficult when the source of the ECG signal is unknown. The strategy for RR intervals in this work is coarse-to-fine detection, and the accurate RR intervals are obtained from the rough RR intervals. The rough RR intervals clearly play a role in the species recognition system, which is combined with a decision tree and clustering algorithm. Indeed, the rough RR intervals can approximate the real RR intervals and serve as a good feature for species recognition (see Fig. 4).To the best of our knowledge, this method has not been proposed before.

There are limitations and insufficiency in this work. First, the evaluation of the algorithm performance for detecting the HR of animals is not sufficient. There are databases, i.e., the MIT-BIH database, for human HR detection with references, but a standard ECG database of animals for reference and comparison is still lacking. Despite this lack of reference data, observations show that qualitative evaluation based on experimental data is feasible. Second, in the species recognition step, to develop a relatively fast procedure, only two features are selected. While taking into account the efficiency, more suitable features should be considered to increase the accuracy. Third, the error in species recognition would influence the accuracy of HR detection. For example, a selected time interval of detection that is larger than a suitable time interval will lead to missed detection. Last, but not least, the width of the sliding window in the improved PT algorithm is set by empirical values (see Table 1). However, these empirical values can be further adjusted by researching additional data to improve the HR detection. In addition, adjusting the sliding window automatically based on previous results rather than empirical values would be an improvement.

\section{Conclusion}

We have proposed a novel method based on the standard PT algorithm to detect the HR of previously unknown species. The principle of the method is using the results of species recognition to adjust the parameters in the PT algorithm for HR detection in real time. The species recognition is achieved by using a decision tree for classification in which two features are extracted from the processed ECG signal segments and are used as training and testing data. Without prior information about the test subjects, the method can detect the HR of rats, mice, frogs, humans and rabbits with high accuracy. In particular, by using the proposed algorithm to test some ECG signals of humans from the MIT-BIH database, the average accuracy rate can reach $99.71 \%$. In future work, the application of the HR detection method to more species can be investigated.

\section{Conflict of Interest}

The authors declare that there are no conflicts of interest regarding the publication of this paper. 


\section{Acknowledgment}

This work is supported by the 13th Five-Year Plan of the National Educational Science Key Issues of the Ministry of Education in 2017 under Grant No. DLA170428.

\section{References}

[1] Noujaim, S. F., Lucca, E., Munoz, V., Persaud, D., Berenfeld, O., Meijler, F. L., \& Jalife, J. (2004). From mouse to whale: A universal scaling relation for the PR Interval of the electrocardiogram of mammals. Circulation, 110(18), 2802-2808.

[2] Zhang, G. Q., \& Zhang, W. (2009). Heart rate, lifespan, and mortality risk. Ageing Research Reviews, 8(1), 52-60.

[3] Fox, K., Borer, J. S., Camm, A. J., Danchin, N., Ferrari, R., Sendon, J. L. L., Steg, P. G., Tardif, J., Tavazzi, L., \& Tendera, M. (2007). Resting heart rate in cardiovascular disease. Journal of the American College of Cardiology, 50(9), 823-830.

[4] Wannamethee, G., Shaper, A. G., \& Macfarlane, P. W. (1993). Heart rate, physical activity, and mortality from cancer and other noncardiovascular diseases. American Journal of Epidemiology, 137(7), 735-748.

[5] Kranjec, J., Begus, S., Drnovsek, J., \& Gersak, G. (2014). Novel methods for noncontact heart rate Measurement: A Feasibility Study. IEEE Transactions on Instrumentation \& Measurement, 63(4), 838-847.

[6] Li, X., Chen, J., Zhao, G., \& Matti, P. (2014). Remote heart rate measurement from face videos under realistic situations. Proceedings of the 2014 IEEE Conference on Computer Vision and Pattern Recognition (pp. 4264-4271).

[7] Takano, C., \& Ohta, Y. (2007). Heart rate measurement based on a time-lapse image. Medical Engineering \& Physics, 29(8), 853-857.

[8] Hamilton, P. S., \& Tompkins, W. J. (1986). Quantitative investigation of QRS detection rules using the MIT/BIH arrhythmia database. IEEE Transactions on Biomedical Engineering, 33(12), 1157-1165.

[9] Vullings, R., Vries, B., \& Bergmans, J. W. M. (2011). An adaptive Kalman filter for ECG signal enhancement. IEEE Transactions on Biomedical Engineering, 58(4), 1094-1103.

[10] Borjesson, P. O., Pahlm, O., Sornmo, L., \& Nygards, M. E. (1982). Adaptive QRS detection based on maximum a posteriori estimation. IEEE Transactions on Biomedical Engineering, 29(5), 341-351.

[11] Pan, J., \& Tompkins, W. J. (1985). A real-time QRS detection algorithm. IEEE Transactions on Biomedical Engineering, 32(3), 230-236.

[12] Ahlstrom, M. L., \& Tompkins, W. J. (1983). Automated high-speed analysis of holter tapes with microcomputers. IEEE Transactions on Biomedical Engineering, 30, 651-657.

[13] Fraden, J., \& Neumann, M. R. (1980). QRS wave detection. Medical Biological Engineering \& Computing, $18(2), 125-132$.

[14] Kadambe, S., Murray, R., \& Boudreaux-Bartels, G. F. (1999). Wavelet transform-based QRS complex detector. IEEE Transactions on Biomedical Engineering, 46(7), 838-848.

[15] Gyaw, T. A., \& Ray, S. R. (1994). The wavelet transform as a tool for recognition of biosignals. Biomedical Sciences Instrumentation, 30, 63-68.

[16] Dokur, Z., Olmez, T., Yazgan, E., \& Ersoy, O. K. (1997). Detection of ECG waveforms by neural networks. Medical Engineering \& Physics, 19(8), 738-741.

[17] Xue, Q., Hu, Y. H., \& Tompkins, W. J. (1992). Neural-network-based adaptive matched filtering for QRS detection. IEEE Transactions on Biomedical Engineering, 39(4), 317-329.

[18] Kohler, B. U., Hennig, C., \& Orglmeister, R. (2002). The principles of software QRS detection. IEEE Engineering in Medicine \& Biology Magazine the Quarterly Magazine of the Engineering in Medicine \& 
Biology Scoiety, 21(1), 42-57.

[19] Neuvo, Y., Rajan, G., \& Mitra, S. (1987). Design of narrow-band FIR bandpass digital filters with reduced arithmetic complexity. IEEE Transactions on Circuits \& Systems, 34(4), 409-419.

[20] Goovaerts, H. G., Ros, H. H., Van, D. A. T. J., \& Schneider, H. (1976). A digital QRS detector based on the principle of contour limiting. IEEE Transactions on Biomedical Engineering, 23(2), 154-160.

[21] Thakor, N. V., Webster, J. G., \& Tompkins, W. J. (1983). Optimal QRS detector. Medical \& Biological Engineering \& Computing, 21(3), 343-350.

[22] Laguna, P. (1992). Adaptive filtering of ECG baseline wander. Proceedings of the International Conference of the IEEE Engineering in Medicine \& Biology Society. IEEE.

[23] Thakor, N. V., \& Zhu, Y. S. (1991). Applications of adaptive filtering to ECG analysis: Noise cancellation and arrhythmia detection. IEEE Transactions on Biomedical Engineering, 38(8), 785-794.

[24] Christov, I. (2004). Real time electrocardiogram QRS detection using combined adaptive threshold. Biomedical Engineering Online, 3(1), 1-9.

[25] Li, C., Zheng, C., \& Tai, C. (1995). Detection of ECG characteristic points using wavelet transforms. IEEE Transactions on Biomedical Engineering, 42(1), 21-28.

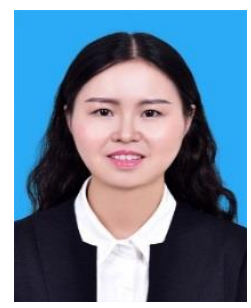

Dan Li received a B.S. degree in electronic information engineering from Xijing University, China, in 2014, and an M.S. degree in computer science from Guizhou Normal University in 2017. She currently works as an algorithm engineer in Chengdu Techman Software Co. LTD. Her current research interests include signal acquisition systems research and the application of machine learning algorithms.

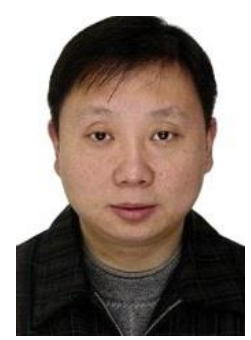

Wu Huang received a B.S. degree in computer science from Sichuan University, China, in 1993, and an M.S. degree in computer science from Sichuan University in 2000. He is currently pursuing a Ph.D. degree in the School of Computer Science at Sichuan University. He is a lecturer in the School of Computer Science at Sichuan University, China. His research interests include embedded real-time systems and intelligent medical instruments.

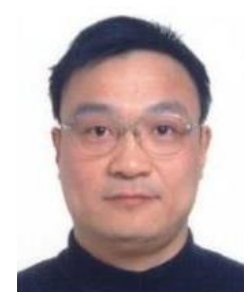

Guobiao Xu received a B.S. in computer science from Sichuan University, China, in 1990, and he received an M.S. in computer science from Sichuan University, China in 1998. Currently, he is an associate professor in the School of Computer Science at the Civil Aviation Flight University of China. His research interests are computer graphics and CAI.

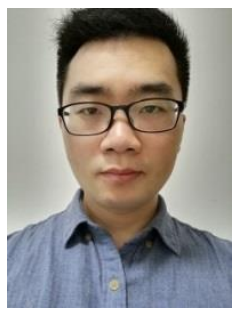

Tao Zhang received a B.S. degree in automation from Southwest University of Science and Technology, China, in 2009, and the M.S. degree in optics from University of Electronic Science and Technology of China, China, in 2017. He currently works as an algorithm engineer in Chengdu Techman Software Co. LTD., China. His professional research interests include machine learning in health care and biological signal processing. 
Zhonghui Jiang received a B.S. degree in mechanical engineering from Southwest Jiaotong University, China, in 2015, and an M.S. degree in vehicle operation engineering from State Key Laboratory of Traction Power, Southwest Jiaotong University, China, in 2018. He currently works as an algorithm engineer in Chengdu Techman Software Co. LTD., China. His professional research interests are in data processing and analysis and research and application of machine learning algorithm.

Defu Cheng received a B.S. degree in electronic and information engineering from southwest University, China, in 2006. He received the M.S. degrees in electronic and information engineering from Southwest University, China, in 2009. From 2009 to 2019, He is currently a hardware engineer of research department in Chengdu Techman Software Co. LTD., China. His current research interests include smart wearable, signal acquisition system and medical devices. 